
\title{
pmaching

Titre : Les impacts potentiels des communications médiatiques sur la santé psychologique des policiers québécois au travail : Étude exploratoire

Rubrique : Article de recherche

\section{Auteur(s)}

$1:$ Kimberley D. Gagnon

2: Andrée-Ann Deschênes

3 : Josée Laflamme

Citation : D. Gagnon, K., Deschênes, A.-A. et Laflamme, J. (2020). Les impacts potentiels des communications médiatiques sur la santé psychologique des policiers québécois au travail : Étude exploratoire. Ad Machina, 4(1). doi: 10.1522/radm.no4.1246
Affiliation des auteurs
1 : Université : Université du Québec à Rimouski
2 : Université : Université du Québec à Rimouski
3 : Université : Université du Québec à Rimouski
Courriel : kimberley.deroygagnon@uqar.ca
Courriel : andree-ann_deschenes@uqar.ca
Courriel : josee_laflamme@uqar.ca

\section{Remerciements}

\section{Déclaration des conflits d'intérêts}

\ Aucun conflit d'intérêts à déclarer

Conflit d'intérêts à déclarer (veuillez détailler)

Détails : 


\title{
Résumé (250 mots)
}

Les policiers exercent un métier où ils sont confrontés à des situations graves et complexes. Plus encore, ils jouent un rôle central et d'une grande importance dans la société; ils assurent la sécurité publique et agissent à titre d'intervenants de première ligne (Leclercq, 2008; Shane, 2010). La presse négative combinée à l'arrivée des nouvelles technologies et leur grande accessibilité représente un enjeu supplémentaire qui peut affecter la santé psychologique des policiers. Depuis quelques années, les policiers font régulièrement la manchette, souvent de manière négative. La médiatisation de ces événements a-t-elle exercé un impact négatif sur la santé psychologique au travail des policiers concernés ? L'étude de Chermak et al. (2006) soutient que les médias représentent souvent la source centrale de perception de la légitimité policière pour les citoyens. La présente étude vise à explorer la relation entre les communications médiatiques et la santé psychologique des policiers. Elle entend utiliser le modèle théorique de Gilbert, Dagenais-Desmarais et Savoie (2011) pour expliquer la santé psychologique au travail. Les communications médiatiques seront discutées selon le modèle général des effets réciproques de la couverture médiatique de Kepplinger (2007). En considérant ce qui précède et puisqu'il s'agit d'une étude qualitative, un échantillonnage par réseau a été réalisé pour recruter les participants. Au total, 12 policiers ont accepté de participer, sur une base volontaire, à une entrevue semi-dirigée. Cette recherche a permis de reconnaître l'influence des communications médiatiques tant sur la santé psychologique des policiers au travail (désengagement, remise en question, consommation excessive de psychotropes, méfiance, état dépressif et perte d'intégrité pour n'en nommer que quelques-uns) que sur leur réseau social de même que sur l'opinion des citoyens envers eux.

\begin{abstract}
Law enforcement is a profession where individuals are faced with serious and complex situations. More importantly, they play a pivotal role in our modern society; they provide public safety and act as frontline during crisis (Leclercq, 2008; Shane, 2010). The arrival of new technologies, negative press brought forward through a near infinite number of media outlet and their wide accessibility causes issues that can easily affect the psychological bealth of a police officer. In recent years, law enforcement has been regularly breaking headlines, often in a negative way. Ergo, does the coverage of these events affect the psychological health at work of the police officers concerned? The study by Chermake and al. (2006) upholds that the media are often the central source of citizens' perception of police legitimacy. In light of these findings, the present study is intended to understand the relationship between media communications of police events and their psychological health. This study intends to use the theoretical model of Gilbert, Dagenais-Desmarais and Savoie (2011) to explain psychological health at work. Media communications will be discussed according to the general model of the reciprocal effects of media coverage by Kepplinger (2007). Considering the above, and since this is a qualitative study, network sampling was used to select the participants; twelve (12) police officers bave accepted to participate in this study on a voluntary basis via a semi-structured interview. This research allowed to recognize the influence of media communications on the psychological health and well-being of law enforcement officers at work (such as loss of commitment in the workplace and at home, questioning one's abilities, excessive use of psychoactive drugs, mistrust, depressive state and loss of integrity) as well as on social media and in citizen opinion about them in general.
\end{abstract}

\section{Mots clés}

Policier, santé psychologique au travail, communication médiatique, bien-être au travail, détresse au travail, presse négative

\section{Droits d'auteur}

Ce document est en libre accès, ce qui signifie que le lectorat a accès gratuitement à son contenu. Toutefois, cette œuvre est mise à disposition selon les termes de la licence Creative Commons Attribution (CC BY NC). 


\title{
Les impacts potentiels des communications médiatiques sur la santé psychologique des policiers québécois au travail : étude exploratoire
}

\author{
Kimberley D. Gagnon \\ Andrée-Ann Deschênes \\ Josée Laflamme
}

\section{Introduction}

Selon l'Institut universitaire en santé mentale de Montréal (IUSMM, 2018), les problèmes de santé mentale font perdre 30 milliards de dollars (coûts indirects inclus) par année aux entreprises canadiennes. Au Québec, un individu sur cinq souffrira de détresse psychologique au cours de sa vie. Ce sont les travailleurs qui présentent un plus grand risque d'être touché par cette détresse : en effet, environ $20 \%$ de la population active aura à faire face à un problème de santé directement lié au stress. Le Mouvement santé mentale Québec (2014) dénote que sur les 10 principales causes d'invalidité (inaptitude au travail), 5 sont attribuables à des troubles d'ordre psychologique. Au Canada, 500000 personnes s'absentent tous les jours du travail en raison de problèmes de santé psychologique, ce qui engendre d'énormes coûts pour les organisations.

Des études ont déterminé que certains emplois étaient plus susceptibles que d'autres de générer des problèmes de santé mentale (Cyr, 2010; Marchand et al., 2005; Tennant, 2001). En ce sens, plusieurs recherches ont tenté de vérifier si cette observation était représentative du travail des policiers (Collins et Gibbs, 2003; Cyr, 2010; Deschamps, Paganon-Badinier, Marchand et Merle, 2003; Ellrich et Baier, 2015; Renck, Weisæth et Skarbö, 2002; Théroux, 2000). Il a été démontré que ceux-ci étaient exposés à des situations stressantes qui peuvent potentiellement engendrer des manifestations de détresse psychologique (Burke, 1993; Carlier, Lamberts et Gersons, 2000; Collins et Gibbs, 2003; Cyr, 2010; Ellrich et Baier, 2015; Kopel et Friedman, 1997; Renck et al., 2002; Stephens, Long et Miller, 1997).

Rappelons que les policiers jouent un rôle central et d'une grande importance dans la société : ils assurent la sécurité publique et agissent à titre d'intervenants de première ligne (Leclercq, 2008; Shane, 2010). Selon Regehr, Johanis, Dimitropoulos, Bartram et Hope (2003), les policiers exercent deux rôles : d'un côté, ils font figure d'autorité, échangent avec le public et s'assurent que les gens sont en sécurité (mandat social); de l'autre, ils travaillent auprès de gens qui représentent un danger pour la société, sont présents sur les lieux de crime et se retrouvent parfois devant des situations incontrôlables et imprévisibles où leur vie et celle des autres peuvent être en danger (mandat légal). Ce double mandat complexifie leur travail. Malgré tout, ils sont tenus d'agir de manière rapide et réfléchie. Les policiers doivent intervenir promptement dans l'imprévisibilité et l'urgence. Outre ces facteurs opérationnels, les policiers doivent aussi composer avec des particularités organisationnelles : horaire inhabituel, «hiérarchie de style paramilitaire », négativisme des médias et du public (Desjardins, 2018; De Soir, Daubechies et Van den Steene, 2012).

D’ailleurs, les 11 policiers qui ont participé à l'étude de Regehr et al. (2003) ont connu un événement traumatisant largement publicisé. L'enquête publique a entraîné de nombreuses conséquences pour les policiers, notamment le stress chronique, l'incertitude à l'égard du processus, l'impact sur la réputation, les rumeurs au travail, un sentiment de résignation, l'impression de ne pas pouvoir entrevoir d'issue positive, etc. Cette étude a établi que tous les policiers avaient subi les conséquences de l'enquête publique les visant et que c'est cet événement qui aurait provoqué le plus de stress durant leur carrière (De Angelis et Wolf, 2013; Regehr et al., 
2003; Sundaram et Kumaran, 2012). En somme, une couverture médiatique peut également exercer une influence sur le degré de stress des policiers en exercice.

\section{Contexte théorique}

\subsection{La santé psychologique au travail}

Nombreuses sont les études qui présentent le bien-être psychologique et la détresse psychologique comme les deux principaux aspects de la santé mentale; l'un positif et l'autre négatif (Deschênes et al., 2018; Gilbert et al., 2011; Morin, 2008; Savoie, Brunet, Boudrias et Gilbert, 2010). Deschênes et al. (2018) ainsi que Gilbert et al. (2011) énoncent qu'il ne s'agit pas de composantes qui se situent à l'une ou l'autre des extrémités d'un pôle, mais plutôt de dimensions qui font partie d'un continuum. La santé mentale ne signifie pas nécessairement qu'il y a absence de symptômes négatifs, comme le désengagement social, l'agressivité/irritabilité, l'anxiété/dépression ou l'autodévalorisation, mais un équilibre entre l'engagement social, le bonheur, la sociabilité, l'estime de soi, le contrôle de soi et les événements du quotidien. Un sentiment de bien-être élevé chez un individu ne signifie pas impérativement un faible degré de détresse psychologique. Il s'agit de deux composantes différentes, mais interdépendantes (Deschênes et al., 2018; Massé et al., 1998). Par conséquent, la santé psychologique doit être considérée comme une conception bidimensionnelle : l'absence de maladie, mais aussi l'existence d'aspects positifs (Bruchon-Schweitzer et Boujut, 2002; Desjardins, 2018).

En considérant ce qui précède, le présent article entend employer le modèle théorique de Gilbert et al. (2011). Il représente un construit global qui se décompose en deux aspects : le bien-être psychologique au travail (BEPT) et la détresse psychologique au travail (DPT). Le bien-être psychologique au travail repose sur les facteurs de sérénité, d'engagement au travail et d'harmonie sociale. Plus précisément, l'individu qui ressent un sentiment de bien-être psychologique au travail témoigne d'un équilibre émotionnel, d'un désir de prendre soin de ses relations sociales avec ses collègues. Il démontre aussi de l'implication, de l'engagement, de la motivation et de l'ambition dans l'accomplissement de ses tâches. Les facteurs de détresse psychologique au travail, sont l'anxiété/dépression, l'irritabilité/agressivité et le désengagement au travail. L'individu peut se sentir stressé, sous pression, triste, inutile, irritable, présenter une difficulté à bien gérer ses problèmes, démontrer de l'impatience avec autrui ou encore paraitre démotivé à l'égard de son travail et dans l'amorce de nouveaux projets (Deschênes et al., 2018; Gilbert et al., 2011).

\subsection{Les communications médiatiques}

Depuis quelques années, les communications médiatiques occupent une place croissante dans la vie des individus. Les médias offrent à ceux-ci la possibilité de partager, de participer, de communiquer et de s'informer sur leur milieu (Lin, Lachlan, Spence et Sellnow, 2016). Les médias de masse s'avèrent certainement un moyen efficace d'avoir accès à de nombreuses informations rapidement. Les gens consomment les nouvelles sur plusieurs plateformes: Internet (qui inclut, entre autres, les sites de nouvelles et les réseaux sociaux), la télévision, la radio et les journaux (Chermak, McGarrell et Gruenewald, 2006; Graziano, Schuck et Martin, 2010; Kepplinger, 2007; Kepplinger et Glaab, 2007; Kepplinger et Zerback, 2012; Lin et al., 2016; Miller, Davis, Henderson, Markovic et Ortiz, 2005; Vasterman, 2005; Vasterman, Yzermans et Dirkzwager, 2005).

Aussi populaires soient-ils, les médias de masse sont souvent pointés du doigt par le public. Cependant, ils demeurent une source importante de contenu et d'information en offrant un flux continuel d'information. Toutefois, l'accumulation et la surcharge d'informations en sont des effets pervers potentiels (Chermak et al., 2006; Graziano et al., 2010; Kepplinger et Glaab, 2007; Kepplinger et Zerback, 2012; Lin et al., 2016; Miller et al., 2005; Vasterman, 2005; Vasterman et al., 2005). 
Plus spécifiquement, les médias sociaux peuvent s'avérer des sources d'information pour la population (Chermak et al., 2006; Graziano et al., 2010; Kepplinger et Glaab, 2007; Kepplinger et Zerback, 2012; Lin et al., 2016; Miller et al., 2005; Vasterman, 2005; Vasterman et al., 2005). Toutefois, ce type de plateforme n'assure pas qu'un contenu fiable et crédible, surtout lors de situations de crise. L'abondance et la facilité d'accès à cette information incitent les individus à s'engager dans un traitement heuristique avec un faible effort cognitif plutôt que d'analyser et de systématiser ce contenu. Autrement dit, il est possible qu'un individu partage des informations sur un événement sur ses comptes de médias sociaux sans se demander s'il s'agit d'informations vérifiées. Les informations inexactes ou incomplètes peuvent engendrer des rumeurs. Ces rumeurs sont une conséquence du besoin des individus d'être informés et de connaittre la progression d'un événement. La plupart du temps, elles se manifestent lorsque les sources d'information ne sont pas en mesure de communiquer les faits souhaités assez rapidement au public. Cela s'explique par le fait que les individus ont tendance à percevoir la mise à jour rapide des informations comme plus pertinentes telles que les nouvelles « dernière heure » (Chermak et al., 2006; Graziano et al., 2010; Miller et al., 2005). Pourtant, si la mise à jour de ces informations est maladroitement exécutée, il est probable que le public critique sa qualité (Lin et al., 2016). Lin et al. (2016) ajoutent que l'accessibilité et la transmission des informations ont un impact sur la gestion de crises.

Il va de soi que les journalistes ont un rôle à jouer : après tout, ce sont eux qui « construisent » la nouvelle. La construction qu'en font les médias de masse a un impact sur la perception du public, ce qui influence les relations entre les citoyens et les policiers (Chermak et al., 2006; Graziano et al., 2010; Miller et al., 2005). D'autant plus que depuis quelques années, les policiers font régulièrement la manchette, souvent de manière négative, comme dans le cas de l'agente Stéfanie Trudeau (mieux connue sous le quolibet « Matricule 728 »), des dénonciations d'abus envers les Autochtones, des interventions musclées, etc. Or, ces événements semblent avoir exercé un impact négatif sur leur santé psychologique.

Cet article entend utiliser le modèle de Kepplinger (2007) qui analyse les effets réciproques des médias de masse sur les individus exposés dans des reportages médiatiques. L'auteur présente un schéma de rétroaction avec trois ensembles de variables: (1) la couverture médiatique en tant qu'institution; (2) la sensibilité de même que le traitement des informations; (3) les effets observables sur les sujets. Ce modèle s'appuie tant sur la théorie expliquant les effets et la prévalence des médias sur la société que sur des données empiriques (Kepplinger, 2007). Toutefois, il sera adapté au contexte du travail des policiers, venant ainsi bonifier les travaux de Korn et Einwiller (2013) sur l'effet d'une couverture médiatique d'une organisation sur les employés, ainsi que ceux de Regehr et al. (2003) sur les effets d'une enquête publique sur les policiers qui ont vécu un événement traumatisant en milieu de travail.

\section{L'objectif}

Malgré le contexte particulier du travail des policiers et de la nature des médias de masse, l'absence d'information à ce sujet ne permet toutefois pas de mobiliser les connaissances préexistantes pour répondre à ce questionnement (St-Denis, 2018), à savoir si les communications médiatiques ont une influence sur la santé psychologique des policiers au travail. De manière plus précise, les données actuelles sont insuffisantes, d'où l'intérêt d'explorer l'existence d'un lien entre la santé psychologique des policiers québécois et les communications médiatiques. De plus, il ne semble pas y avoir d'étude préalable qui touche aux thèmes principaux de cette recherche.

À partir de ce constat et de la littérature limitée, cet article a comme objectif de comprendre comment les communications médiatiques ont un impact sur la santé psychologique des policiers au travail. En raison des informations théoriques précédentes, la question suivante se pose : comment la couverture médiatique négative influence la santé psychologique au travail des policiers? 


\section{La méthodologie}

\subsection{Le devis de recherche}

Cette étude de nature qualitative a pour objectif d'explorer les impacts potentiels des communications médiatiques sur la santé psychologique des policiers au travail. En raison des informations théoriques existantes, la question suivante se pose : comment les communications médiatiques impliquant des policiers influencent-elles leur santé psychologique au travail?

Pour répondre à cette question, les approches privilégiées sont celles de la recherche documentaire (articles scientifiques, littérature, rapports de recherche) en plus d'entrevues semi-dirigées afin de laisser les participants s'exprimer en se référant à leurs expériences uniques. C'est alors que cette approche exploratoire, la démarche qualitative, met l'accent sur les participants qui évoluent dans leur environnement habituel avec l'optique de comprendre les nuances qui décrivent les phénomènes psychosociaux qui sont associés à leurs expériences (Bouchard et Cyr, 2005; Dumez, 2011; Fortin et Gagnon, 2016; Gallant, 2018). Bref, étant donné que l'entrevue semi-dirigée a été retenue comme méthode de collecte de données et qu'elle a été construite à l'aide de la revue de littérature, la grille d'entrevue permet de saisir les thèmes saillants des recherches préexistantes afin de les aborder avec les participants.

\subsection{La population à l'étude}

Un échantillonnage par réseau a été réalisé pour recruter les participants. Les critères de sélection et d'inclusion sont les suivants : être un policier actuellement en service au Québec, être en contact avec les citoyens sur une base quotidienne et avoir été exposé à un épisode médiatisé. Comme la participation volontaire est privilégiée, le nombre de femmes et d'hommes n'est pas un critère primaire. La visée de l'étude est de mettre en lumière l'impact des communications médiatiques sur la santé psychologique au travail des policiers et non de faire une comparaison entre les sexes. Une attention particulière est portée à l'échantillon visé qui inclut une représentation des différents milieux et organisations policières autant municipales que provinciales.

Pour ce qui est de la collecte de données, Fortin et Gagnon (2016) soutiennent que «le nombre de participants à une étude qualitative n'est pas décidé d'avance, car la taille de l'échantillon dépend des données recueillies» (p. 31). En considérant les propos précédents, cette recherche dénombre ainsi la collaboration de 12 policiers et policières d'organisations provinciales et municipales du Québec. Ils ont tous accepté de participer à la recherche sur une base volontaire. Le tableau 1 qui suit fait brièvement leur présentation : 
Tableau 1

Présentation des participants de l'étude

\begin{tabular}{|c|c|c|c|c|c|}
\hline $\begin{array}{l}\text { Numéro du participants } \\
\text { ou de la participante }\end{array}$ & Âge & Expérience & Fonction & $\begin{array}{c}\text { Date de } \\
\text { l'entrevue }\end{array}$ & $\begin{array}{l}\text { Durée de } \\
\text { l'entrevue }\end{array}$ \\
\hline Participant $n^{\circ} 1$ & 52 ans & 27 ans & Officier & 14 mai 2019 & 50 minutes \\
\hline Participant $\mathrm{n}^{\circ} 2$ & 32 ans & Plus de 6 ans & Superviseur & 30 mai 2019 & 35 minutes \\
\hline Participant $n^{\circ} 3$ & 45 ans & 20 ans & Superviseur & 29 juillet 2019 & 93 minutes \\
\hline Participant $n^{\circ} 4$ & 46 ans & 23 ans & Officier & 18 janvier 2020 & 36 minutes \\
\hline Participant $n^{\circ} 5$ & 32 ans & 8 ans & Superviseur & 18 janvier 2020 & 51 minutes \\
\hline Participante $n^{\circ} 6$ & 36 ans & 12 ans & Patrouilleuse & 11 février 2020 & 53 minutes \\
\hline Participant $\mathrm{n}^{\circ} 7$ & 33 ans & 13 ans & Superviseur & 12 février 2020 & 35 minutes \\
\hline Participant $n^{\circ} 8$ & 38 ans & 14 ans & Officier & 4 mars 2020 & 28 minutes \\
\hline Participante $n^{\circ} 9$ & 39 ans & 18 ans & Lieutenante détective & 5 mars 2020 & 24 minutes \\
\hline Participante $n^{\circ} 10$ & 35 ans & 12 ans & Superviseur & 11 mars 2020 & 27 minutes \\
\hline Participant $n^{\circ} 11$ & 41 ans & 15 ans & Sergent & 31 mars 2020 & 30 minutes \\
\hline Participant $n^{\circ} 12$ & 50 ans & 20 ans & $\begin{array}{c}\text { Agent aux relations } \\
\text { publiques }\end{array}$ & 28 avril 2020 & 39 minutes \\
\hline
\end{tabular}

\subsection{L'instrument de mesure}

L'entrevue semi-dirigée, privilégiée dans le cadre de cette étude, se caractérise par son approche qui oriente ses questions en se basant sur une liste de thèmes préétablis (Fortin et Gagnon, 2016). Imbert (2010) propose que l'objectif de ce type d'entrevue est de « saisir le sens d'un phénomène complexe tel qu'il est perçu par les participants et le chercheur dans une dynamique de co-construction du sens » (p. 25; Moisseron, 2019). Le guide d'entrevue se présente donc comme un plan dans lequel se retrouvent les thèmes à explorer. L'ordre des thèmes abordés s'adaptera au fil de la discussion entre l'intervieweur et le participant (Gavard-Perret, Gotteland, Haon et Jolibert, 2012), suivra ensuite la transcription intégrale de l'entrevue (Fortin et Gagnon, 2016). 
L'entrevue, d'une durée approximative d'une heure, comporte trois sections. La première consiste à recueillir des renseignements généraux sur le policier, comme le sexe, l'âge, l'état matrimonial, le nombre d'années en fonction et le rôle dans l'organisation. La deuxième section aborde le thème de la santé psychologique au travail. Enfin, la troisième section, quant à elle, aborde la perception des policiers sur l'impact des communications médiatiques. Un formulaire de consentement est au préalable signé par le participant et l'intervieweur.

\subsection{Le plan d'analyse des données}

L'analyse de l'information est effectuée avec l'approche d'analyse de contenu thématique de données qualitatives telle que décrite selon Thomas (2006; Blais et Martineau, 2006; Deschênes, Desjardins et Dussault, 2018). L'approche inductive vise à « réduire les données brutes pour en arriver à extraire le sens derrière ces données. Elle est définie comme un ensemble de procédures systématiques permettant de traiter des données qualitatives. Ces procédures étant essentiellement guidées par les objectifs de recherche » (Blais et Martineau, 2006, p. 15).

Comme suggéré par Blais et Martineau (2006), la méthode d'analyse de contenu thématique comporte quatre étapes. A priori, une fois que les entrevues sont réalisées, celles-ci ont été transcrites textuellement pour en simplifier l'analyse (étape 1 : préparer les données brutes). En réponse à la lecture des entrevues et à la réalisation de résumés, qui illustrent les éléments saillants (étape 2 : faire une lecture attentive et approfondie), vient l'identification de catégories qui comporte les thèmes récurrents en lien avec les objectifs de la recherche (étape 3 : procéder à l'identification et à la description des premières catégories). Et finalement, découle la création d'un modèle qui regroupe les catégories de l'étape précédente (étape 4 : poursuivre la révision et le raffinement des catégories).

C'est ainsi que dans cette recherche, les comptes rendus exhaustifs ont été rédigés à la suite de tous les entretiens. La lecture et la compréhension des propos des participants sont de mise afin de bien saisir leur message. L'analyse des comptes rendus permet de mettre en lumière des thèmes communs à plusieurs des participants. Il s'agit là de la première analyse de l’identification des catégories générales d'analyse du contenu thématique. Suivra la catégorisation des thèmes qui vont être analysés par les affirmations des participants de même qu'être appuyés par la littérature existante permettant ainsi leur validation ou leur réfutation. L'analyse thématique est donc un exercice de réduction et de synthèse des données. L'objectif de cette étude n'est pas le développement ni la confirmation ou la réfutation d'une théorie, mais bien de saisir l'expérience unique des participants pour ainsi relever des thèmes communs à leur expérience.

\section{Les résultats}

Les résultats issus de l'analyse de contenu thématique proposent plusieurs thèmes. Ces thèmes sont exposés dans le tableau 2 qui présente ainsi la première analyse de l'identification des catégories générales d'analyse de contenu thématique. 
Tableau 2

Première analyse - Catégories générales de contenu thématique

\begin{tabular}{ll}
\hline \multicolumn{1}{c}{ Communications médiatiques informationnelles au grand public } \\
\hline \multicolumn{1}{c}{ Thèmes } \\
1. Famille & 12. Peur du jugement \\
2. Diminution de la confiance à l'égard de la & 13. Culpabilité \\
police/Négativisme du public & 14. Consommation excessive \\
3. Financier/Peur de perdre leur emploi & 15. Colère \\
4. Méfiance & 16. Irritabilité \\
5. Survigilance & 17. Augmentation du niveau de stress \\
6. Désengagement & 18. Vulnérabilité \\
7. Diminution de la motivation & 19. Perte d'intégrité \\
8. Présentéisme & 20. Impuissance \\
9. Isolement & 21. État dépressif \\
10. Solitude & 22. Diminution du sentiment de \\
11. Perte de jouissance sociale & compétence/Remise en question \\
\hline
\end{tabular}

\subsection{Détermination des catégories et des thèmes}

À la suite de cette première étape d'analyse vient le temps de séparer les thèmes en catégories. Toujours à l'aide des propos des participants, les catégories ont été déterminées. Quatre catégories sont ainsi ressorties : les impacts reliés au travail, les impacts reliés aux comportements sociaux, les impacts reliés à soi et les impacts indirects. La figure suivante résume le processus d'analyse des données.

Figure 1

Schéma de l'analyse de contenu thématique

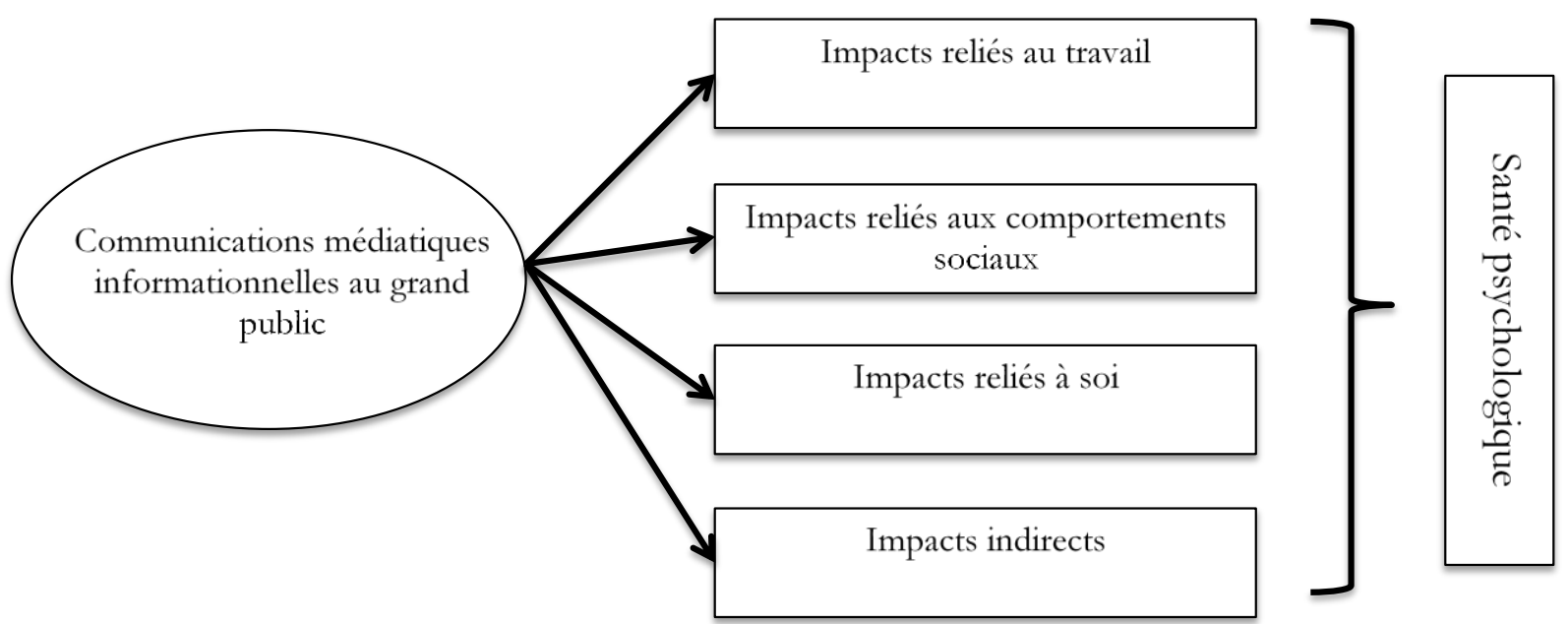


Le tableau 3 regroupe les catégories précitées ainsi que les divers thèmes qui composent chacune d'elles. Ces thèmes sont communs à la majorité des participants. Au total, 19 thèmes sont divisés dans les 4 catégories. Il est à mentionner que certains thèmes ont été rassemblés étant donné leurs similitudes.

Tableau 3

Deuxième analyse - Catégories générales de contenu thématique

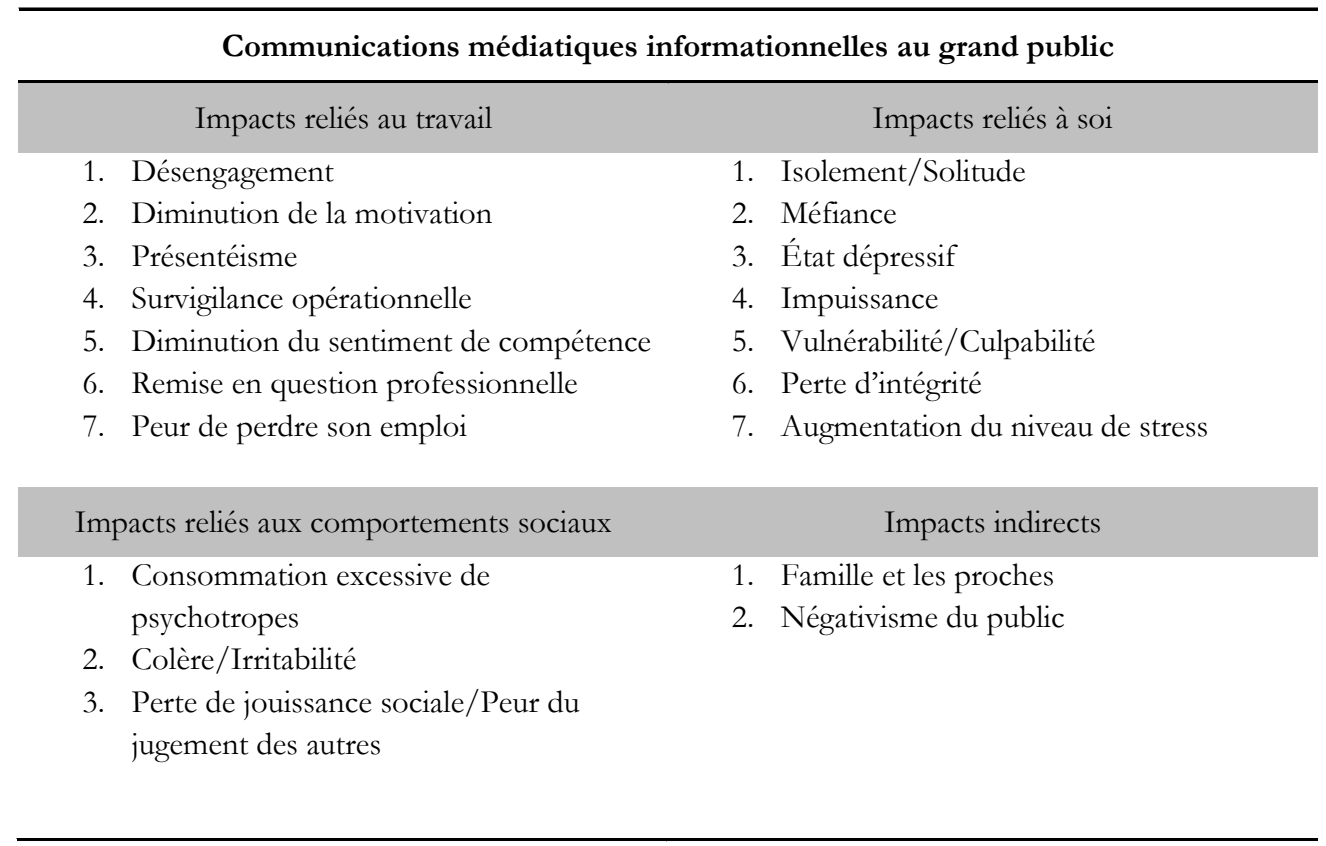

Chaque catégorie avec ses thèmes respectifs va être appuyée et analysée à l'aide des affirmations des policiers. Les thèmes vont être éclairés et expliqués par des exemples.

\subsection{Impacts reliés au travail}

\subsubsection{Désengagement}

Quelques participants ont mentionné qu'à la suite d'un événement négatif hautement médiatisé dans lequel ils ont été impliqués, ils vivent un désintérêt, une absence d'engouement lors du retour au travail. Visiblement, leur bien-être psychologique au travail a décliné à la suite de l'attention médiatique néfaste vécue. À ce propos, le participant $n^{\circ} 1$ mentionne: Je fais mes shifts, j’suis bien payé, ça s'arrête là, pas d'overtime, je fais absolument rien.

\subsubsection{Diminution de la motivation}

Il s'avère intéressant de constater que les participants qui notent un désengagement - une diminution d'implication dans leur environnement professionnel — ont aussi subi une diminution de leur motivation au travail - la perte de volonté de répondre à un besoin ou à un objectif (Mindforest, 2014). En effet, il est possible de percevoir une interdépendance entre ces deux thèmes. De plus, ils notent que la diminution de leur motivation résulte de leur surexposition à une situation qui a fait les manchettes. Le participant $\mathrm{n}^{\circ} 5$ souligne: À un moment donné, c'est sûr qu'il y en a une qui va se dire: "je vais faire mon travail, puis ça ne veut pas vraiment dire que je vais gagner", et puis c'est sûr qu'ily en a qui se disent: "moi je vais faire ma petite job, juste deux semaines et je vais avoir ma paie et puis je ne me ferai pas éccurer nulle part ». 


\subsubsection{Présentéisme}

Certains policiers révèlent que leur présence au sein de leur milieu de travail est constante, peu importe leur état de santé (psychologique ou physique). Ceci dit, même s'ils se présentent au travail, cela ne sous-entend pas qu'ils vont avoir une attitude positive envers leurs collègues. Un policier, le participant $\mathrm{n}^{\circ} 8$, indique : La police de 2020 n'est pas celle des années '80. Le "Be-Policing» fait en sorte que les gens prennent ça moins à ccur. Ils viennent travailler: "Punch in et Punch out».

\subsubsection{Survigilance opérationnelle}

Plusieurs policiers nomment qu'ils ressentent une pression incessante pour réaliser leur travail avec un haut degré de professionnalisme, qu'importe l'intervention. La pression d'être observé, de savoir leurs gestes et leurs actions scrutés sans cesse font en sorte qu'ils ont parfois peur d'agir et craignent des conséquences administratives et judiciaires. Ils sont conscients qu'ils sont tenus d'agir avec célérité en toute circonstance. Le policier ne veut pas nuire à sa carrière ni à son nom, ce qui a pour conséquence qu'il va être plus prudent et s'en tenir à ce qui lui a été enseigné afin de protéger son intégrité tout en protégeant celle d'autrui. Dans ce sens, le participant $\mathrm{n}^{\circ} 12$ révèle : Lors d'une manifestation où on sait que les médias sont attendus. C'est sûr qu'on sait qu'il a de la caméra, on va être un peu plus prudent c'est sûr que ça nous influence, mais ça reste qu'on va tout le temps travailler selon sur ce qui nous est enseigné.

\subsubsection{Diminution du sentiment de compétence}

Quelques policiers mentionnent qu'à la suite d'une intervention médiatisée, ils vivent une grande pression au point où ils en arrivent à se poser de nombreuses questions en lien avec la manière dont ils ont effectué leur travail. Tous ces questionnements peuvent avoir une influence négative sur leur confiance et leur estime personnelle. La participante $n^{\circ} 9$ énonce: Ça fait que, bien tu sais, ça vient jouer assurément sur l'estime, la santé mentale du fait que là tu te dis : "bien là, je suis un bon à rien » ou... c'est sûr que ça a un impact là sur toute la conscience.

\subsubsection{Remise en question professionnelle}

Plusieurs participants soulignent que la répétition d'une nouvelle crée une remise en question chez eux. Les événements gagnent en ampleur et attirent le regard du public sur le policier. Cette attention médiatique soutenue expose le policier au doute quant à son choix de carrière. Les médias ont aussi le pouvoir de semer l'incertitude dans l'esprit du policier au point où il se sent responsable de tout. De ce fait, la participante $n^{\circ} 6$ précise : Tu sais, tu te remets en question. "Tu veux-tu encore faire ça? C'est ça, je veux-tu encore vivre ça? " Parce qu'on s'expose. Ça peut arriver encore une affaire de même. Avoir une prise d'otages. Tu sais, un autre accident. On est tout le temps, tout le temps, tout le temps exposé. On est toujours les responsables de tout... C'est ça. C'est un fardeau que les policiers portent.

\subsubsection{Peur de perdre son emploi}

Toujours selon les participants, à la suite d'une couverture médiatique, il arrive qu'ils se sentent submergés par tout ce nouvel intérêt. Ils ont parfois peur des conséquences allant jusqu'à appréhender s'ils ont toujours un emploi dans les forces policières. Le participant $n^{\circ} 11$ mentionne: Moi, les impacts que ça a eus, je ne vous cacherais pas que pendant les deux semaines là, j'en ai arraché comme je dis. Comme c'est là, à un moment donné je me suis mis à paniquer, à me questionner : "criss, est-ce que je suis encore une bonne police? J'ai-tu encore ma job dans la police?" On parlait d'à peu près toutes les conséquences possibles là : "Il vas-tu y avoir des accusations criminelles? Je vais-tu être suspendu? » Tu sais, même moi, dans mon processus d'intervention dans le comment j'ai intervenu : "est-ce que j'ai fait ça comme il faut? 》 


\subsection{Impacts reliés aux comportements sociaux}

\subsubsection{Consommation excessive de psychotropes}

Certains participants font référence à l'utilisation de psychotropes pour surmonter les difficultés associées à une couverture médiatique négative sur leur santé psychologique. Selon eux, les difficultés sont notamment la durée du processus judiciaire, l'envie d'oublier ainsi que l'isolement. Ces éléments ne sont que quelques exemples de ce qui peut pousser le policier à tomber dans les excès de consommation de psychotropes. La participante $\mathrm{n}^{\circ} 9$ soutient : Ça peut amener, moi je pense, est-ce que...pas nécessairement l'événement juste en soi, mais avec la médiatisation, mais est-ce que ça peut amener à vouloir oublier après? Est-ce que la personne va peut-être être plus portée à, je ne sais pas moi, retrouver un réconfort dans l'alcool ou ces choses-là?

\subsubsection{Colère/Irritabilité}

Les policiers interviewés sont conscients que la consommation et l'avènement des médias sociaux ont une incidence dommageable sur leur image. En effet, ils savent que la perception du public se construit de la nouvelle, de la façon dont les médias présentent les forces policières. Les policiers vivent ainsi une multitude d'émotions négatives qui s'apparentent à une détresse psychologique : énervement, irritation, animosité, exaspération. Le participant $n^{\circ} 5$ relate : Oui, c'est ça, j'aurais remis un peu mon travail en question parce qu'ily aurait eu un mort, mais là de venir me faire insulter par du monde qui ne connaît rien de l'histoire, parce qu'ils ont lu des affaires sur Facebook. Je trouve que, tu sais, j'ai des collègues ça les affecte, parce qu'on voit qu'ils pognent les nerfs ou que ça arrive tu sais, ou c'est arrivé une fois où j'ai dit...

\subsubsection{Perte de jouissance sociale/Peur du jugement des autres}

Plusieurs termes ont été utilisés par les policiers pour décrire leur inconfort de sortir en public en réponse à la médiatisation d'un événement : la peur d'être reconnu, d'être pointé du doigt, d'être stigmatisé, d'être jugé et de porter une étiquette pour n'en nommer que quelques-uns. Comme mentionné dans le thème précédent, les policiers sont au fait que les médias nourrissent leur image négative, entre autres, par les informations inexactes ou incomplètes, l'écart entre la réalité de la situation et ce qu'ils en ont dit. Le policier se retrouve alors pris dans ce tourbillon et en subit les conséquences sur sa santé psychologique, dont sa liberté de sortir dans des lieux publics, le plaisir de vaquer à ses occupations sans avoir peur du jugement. Le participant $\mathrm{n}^{\circ} 4$ révèle : Cette pression-là de dire: "Si je vais quelque part, je vais être reconnu comme policier et je fais attention à ce que je fais, à ce que je dis ».

\subsection{Impacts reliés à soi}

\subsubsection{Isolement/Solitude}

Selon les policiers, celui qui vit une situation qui bénéficie d'une couverture médiatique a tendance à vouloir s'isoler et être seul pour éviter d'entendre de mauvais commentaires. De cette manière, le policier protège son intégrité. D'ailleurs, un policier qui a de bonnes relations avec ses collègues, et qui est intégré au groupe, reçoit leur appui et leur soutien. Au contraire, celui qui est exclu du groupe se voit isolé. Dans ce sens, le participant $\mathrm{n}^{\circ} 1$ indique : Ca dépend si... la police c'est très gang, si ton policier fait partie de la gang, tu vas avoir un soutient un certain temps. Si ton policier ou ta policière ne fait pas partie d'une gang, souvent il va être isolé.

\subsubsection{Méfiance}

L'ensemble des participants souligne que les médias ont eu un impact sur la perception du public envers les policiers. D'un côté, la manipulation des informations et la répétition de la nouvelle nourrissent la méfiance du public; les policiers ont mauvaise presse et l'accent est souvent mis sur le négatif. L'opinion 
du public se construit avec ces reportages et leur confiance envers les policiers diminue et le sentiment de méfiance augmente. Et de l'autre, les policiers vivent un sentiment de méfiance envers les médias. Bien souvent, ils ignorent comment la nouvelle va sortir. Ils se tiennent ainsi sur leur garde et font plus attention à la manière dont ils interviennent. Le participant $n^{\circ} 7$ souligne : Ils ont tellement peur de se faire avoir par nous autres, le lien de confiance est plus dur à avoir. Ce n'est pas tout le monde, mais une partie de la population, le monde qu'on fait affaire avec surtout.

\subsection{3 État dépressif}

Un certain nombre de policiers voient leur santé psychologique se détériorer après une exposition aux médias ou à la suite d'une intervention difficile. Par exemple, ces derniers peuvent vivre le sentiment d'être détruit, une augmentation de certains symptômes du stress post-traumatique (les difficultés de concentration, détachement, hypervigilance, etc.), un état dépressif, une perte de contrôle voire un arrêt de travail. La totalité des éléments précités fait référence à la détresse psychologique. Le participant $\mathrm{n}^{\circ} 8$ rajoute: Mettons que ça t'ouvre, que ça te donne une vision différente des événements et en même temps ça te sensibilise comme gestionnaire à te dire que des fois il y a des policiers de chez nous qui partent en maladie ou qui parlent de syndrome post-traumatique ou qui partent en arrêt de travail, parce qu'ils nous disent que bon, ça été dur tel appel.

\subsubsection{Impuissance}

La majorité des participants précise qu'ils vivent un sentiment d'impuissance; ils ont l'impression de ne pas être capable de faire quoi ce soit pour rétablir la véracité des événements. Les communications médiatiques sont reconnues d'user de sensationnalisme pour ainsi « vendre » la nouvelle. C'est alors qu'une couverture médiatique fausse exerce une influence négative sur la santé psychologique des policiers. Ces derniers vivent une incapacité envers les médias, envers la diffusion inexacte qu'ils font de l'événement. En effet, le participant $\mathrm{n}^{\circ} 10$ soutient: Pendant cette période-là, c'est sûr que tu as au moins l'impression de contribuer, de te réaliser un peu d'une façon professionnelle si je peux m'exprimer de la sorte, mais c'est sûr qu'étant policier c'est une claque en pleine face que tu recois. Dans le sens que si tu es reconnu coupable c'est l'emprisonnement, tu as un procès à subir, ton nom est partout dans les médias. Il tape mon nom sur Google, toute la série tombe. C'est sûr que oui tu as l'impact personnel, mais là aussi tu es confronté à ton rêve professionnel.

\subsubsection{Vulnérabilité/Culpabilité}

D’après les policiers, il arrive qu'à la suite d'un événement médiatisé, ils se sentent plus vulnérables et plus fragiles. Ils ont l'impression de ne pas avoir le contrôle sur ce qui se passe et ils se sentent coupables de la façon dont ils ont agi bien qu'ils aient simplement appliqué ce qu'ils ont appris durant leur formation. Ils se retrouvent à vivre dans le négatif pendant une certaine période même si c'est un métier qui les passionne. Les policiers sont conscients qu'ils ont une profession à risque et ils sont tenus d'agir de manière réfléchie et rapide en toutes circonstances. C'est ainsi qu'ils sont plus susceptibles de rencontrer journellement des événements qui peuvent être difficiles, voire traumatiques, qui nourrissent leur sentiment de vulnérabilité. Le participant $n^{\circ} 3$ relate: Ce que je vois au niveau physique, ces policiers-là sont quasiment finis, là. Sont quasiment totalement détruits. Sur leur corps physique, le mental réflééchit beaucoup. Ca tourne beaucoup. Puis, comme moi dernièrement, il m'est arrivé un événement au travail et puis j'ai fait une syncope. Puis, moi je pensais qu'une syncope c'est le cour qui arrête et le docteur quand je suis entré à l'hôpital, le docteur m'a expliqué que non, non, une syncope c'est ton corps qui arrête, pas nécessairement le cour. Et là ton cerveau a dit que c'est trop et il a décidé d'arrêter. Il a fait un "reset ». [Inaudible]. Puis, il savait tout ce qui s'est passé là, que j'étais en choc post-trauma et tout ça. 


\subsubsection{Perte d'intégrité}

Quelques participants mentionnent qu'ils ont eu l'impression de perdre leur intégrité, qu'ils ont perdu confiance en eux à la suite de la diffusion d'un reportage les concernant dans les médias. Les policiers remettent en doute la manière dont ils sont intervenus et cherchent même à savoir si ce que les médias en ont dit reflète la réalité ou non. L'image des policiers se retrouve donc entre les mains des médias et par le fait même du public. C'est à ce moment que les policiers savent que la manière dont ils se comportent avec le public a une grande importance sur la construction de cette image. La participante $\mathrm{n}^{\circ} 6$ nomme : Pis ça peut toucher notre intégrité [à la suite d'un reportage]. Tu sais, on est des personnes dévouées nous autres, les policiers, puis on laisserait... on laisserait jamais quelqu'un... en danger.

\subsubsection{Augmentation du niveau de stress}

La quasi-totalité des policiers indique qu'ils ont une profession à risque où ils sont plus susceptibles d'être exposés à des situations stressantes ou à des événements qui peuvent être traumatiques. La complexité de leur travail, la médiatisation des événements et la durée des procédures judiciaires semblent avoir un impact négatif sur la santé psychologique des policiers en exercice. C'est alors que les participants interviewés précisent qu'ils peuvent vivre de l'anxiété et de la fragilité, partir en arrêt de travail, et remarquer une augmentation de leur degré de stress. Le participant $\mathrm{n}^{\circ} 11$ précise : Donc, moi, j'ai été arrêté deux semaines, suite à l'événement pour des troubles d'adaptation, parce que là j'étais comme byperstressé et je ne savais pas où me "garrocher", puis, un peu pour me protéger un peu de ça. Donc, oui malbeureusement, les médias, par l'influence qu'ils ont et par la portée qu'ils peuvent avoir, oui ça a eu un impact négatif, ça, c'est sûr et certain.

\subsection{Impacts indirects}

\subsubsection{Famille et les proches}

La presque totalité des participants observe qu'une attention médiatique occasionne de nombreuses conséquences pour eux et pour les membres de leur famille : séparation, déménagement, difficulté à l'école pour les enfants, etc. Autrement dit, par association, une couverture médiatique soutenue affecte négativement aussi bien la famille du policier que le policier lui-même. Un des policiers de l'étude, le

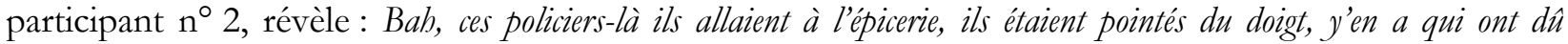
déménager, changer de lieu de travail, parce qu'évidemment leur santé psychologique en prend un coup.

\subsubsection{Négativisme du public}

La plupart des policiers sont conscients que l'attitude du public est influencée par les médias qu'ils consomment. En d'autres mots, la légitimité policière pour les citoyens est basée sur ce qui est véhiculé par les médias. C'est ainsi que les relations entre les citoyens et les policiers semblent se détériorer davantage avec l'image négative diffusée par les communications médiatiques. Inévitablement, c'est la santé psychologique du policier qui en subit les conséquences. À ce propos, le participant $\mathrm{n}^{\circ} 5$ indique : On s'est fait éccurer qu'on ne faisait pas notre job, qu'on était des « sales» et des corrompus, là.

\section{Discussion}

Cet article a voulu explorer le lien entre les communications médiatiques et la santé psychologique des policiers au travail. Lorsqu'un policier fait son entrée dans les forces policières, il fait le serment de protéger autrui. C'est son engagement moral envers le citoyen et son identité professionnelle. Il arrive qu'il puisse perdre le sens de son travail et son engagement moral. En raison de la nature de leur travail et de l'importance de leur rôle dans la société, les policiers souffrent davantage de détresse psychologique causée 
par le stress que la population en général (Violanti, 2010). Il est possible qu'une attention médiatique soutenue représente une difficulté supplémentaire entraînant des conséquences pour le policier. Autrement dit, depuis quelques années déjà, les policiers font couramment la manchette, souvent négativement. Cette attention médiatique amène la population à scruter le travail des policiers et à porter facilement un jugement sur la manière dont ils effectuent leur travail.

Cette étude renforce l'importance de la santé psychologique au travail. Depuis quelque temps, la santé psychologique au travail des intervenants de première ligne semble être un sujet qui est davantage traité dans le milieu de la recherche (Desjardins, 2018). Cependant, les études actuelles sont limitées, voire inexistantes, sur la question que porte ce mémoire. Pourtant, l'arrivée des nouvelles technologies et de leur grande accessibilité combinée avec la presse négative semble être un enjeu additionnel qui peut toucher la santé psychologique au travail des policiers. C'est ainsi que cette étude veut encourager les policiers à protéger leur santé psychologique et souligner les dispositions que les organisations ont mises en place pour aider les policiers, comme le Programme d'aide aux employés (PAE).

Le milieu de l'organisation policière, les collègues et les gestionnaires ont tous un rôle d'une grande importance à jouer lorsqu'un confrère se retrouve malgré lui dans les communications médiatiques. Puisqu'elles sont souvent présentées de manière négative, le soutien et l'appui de son entourage au sein de son milieu sont d'autant plus nécessaires. Le sensationnalisme et la répétitivité de la nouvelle semblent provoquer de nombreuses conséquences autant chez le policier que sur sa famille. Le sentiment de ne pas être seul devant les médias de masse a un effet de baume sur le policier. Cela lui permet de prendre le recul nécessaire pour regarder la situation d'un œil plus objectif. Toutefois, ce n'est pas toujours évident, surtout lorsque le policier se retrouve devant une enquête indépendante. Les policiers vivent beaucoup d'incertitude et d'impuissance en raison de la longue durée de ce processus.

Les policiers se doivent d'agir rapidement en toutes circonstances et ils n'ont pas droit à l'erreur. Lorsqu'ils ne respectent pas ce standard, selon la vision du public et celle des médias, leur santé psychologique en subit ainsi les conséquences. Dans le cadre de leurs fonctions opérationnelles, les policiers peuvent vivre de multitudes événements traumatiques : fusillades, suicides, accidents, meurtres. Les médias contribuent à la création et à l'exploitation de l'événement. Pour le policier impliqué dans ce type d'événement, les médias peuvent participer à sa détresse psychologique en montrant en boucle des images-chocs et des nouvelles liées à l'événement l'impliquant. De plus, le négativisme véhiculé sans cesse par les médias n'aide en rien l'opinion du public envers les policiers; l'image des policiers est teintée négativement par la couverture médiatique. L'ignorance du public quant au rôle du policier gonfle avec ce type de communications médiatiques qui visent le sensationnalisme au détriment des bonnes actions policières.

D’autre part, dans certains cas, les médias peuvent avoir un effet bénéfique sur le travail des policiers. Par exemple, lorsqu'une personne disparait. Les médias et le public sont donc appelés à participer à la recherche et cela favorise donc les chances de retrouver la personne. La collaboration entre les deux est donc empreinte de succès. Les communications médiatiques peuvent avoir de bons côtés, mais souvent, les policiers sont exposés de manière négative; leurs bons coups ne sont que rarement montrés.

D’ailleurs, souvenons-nous, quatre catégories ont été représentées : les impacts reliés au travail, les impacts reliés aux comportements sociaux, les impacts reliés à soi et les impacts indirects. À l'intérieur de ces catégories, 19 thèmes s'y retrouvent. Cette section tente d'établir un lien entre les thèmes identifiés précédemment et les résultats de recherches antérieures représentés par la recension des écrits de cette étude. Quelques extraits de comptes rendus exhaustifs seront identifiés pour valider les propos émis. Toutefois, quelques thèmes ne sont pas ou très peu exprimés par les publications scientifiques, donc ils ne se retrouvent pas à l'intérieur de ce chapitre. C'est ainsi que 13 thèmes sont considérés. 
À titre indicatif, les thèmes absents sont les suivants: le désengagement, la remise en question professionnelle, la peur de perdre son emploi (les impacts reliés au travail); la colère et l'irritabilité de même que la perte de jouissance sociale et la peur du jugement des autres (les impacts reliés aux comportements sociaux); l'isolement et la solitude (les impacts reliés à soi). Pour tenter d'expliquer l'absence de ces thèmes dans la présente étude, il faut prendre en considération plusieurs facteurs, le plus important étant le vide littéraire autour de ces derniers. Il est difficile de dire avec exactitude pour quelles raisons ces thèmes ne font pas ou très peu partie des recherches passées et actuelles. Nul doute que la présente étude révèle une lacune quant aux notions qu'il serait tout aussi nécessaire de prendre en compte. La complexité de la profession de policier, les effets directs et indirects des médias sur les individus ne sont jamais causés par une seule source ni l'influence du comportement social (Kepplinger, 2007). Autrement dit, une combinaison de plusieurs facteurs doit être prise en considération dans une tentative de comprendre et d'expliquer cette constatation. Dans l'ensemble, les prochaines études devront accorder une attention particulière à la limite décrite ci-dessus.

\section{Conclusion}

Cette étude a su démontrer que le policier peut vivre des conséquences psychologiques influencées par la manière dont les médias semblent traiter les informations de nos jours. Toutefois, elle ne répond pas à la question suivante : comment améliorer la collaboration entre les organisations policières et les médias afin de protéger l'intégrité de ces derniers et limiter l'impact des nouvelles instantanées? À l'époque de la popularité des médias imprimés, les policiers vivaient-ils autant avec ce jugement défavorable? Les policiers ont le devoir de protéger l'intégrité d'autrui, de s'abstenir de partager des faits qui pourraient compromettre une enquête. Mais qui protège le policier? Quel est le rôle de l'organisation policière? Cette dernière soutient-elle ses membres? L'organisation doit-elle prendre une plus grande place pour que le policier se sente valorisé, encouragé et soutenu afin de diminuer son niveau de détresse psychologique? Est-ce qu'au cours de la formation des nouvelles recrues, la portée que peuvent prendre les médias est soulevée? Plusieurs questions demeurent sans réponses. Il apparaît donc approprié de poursuivre les recherches afin de mieux comprendre la complexité de la réalité policière au Québec en 2020, mais de surcroît celle des journalistes et leur interrelation.

\section{RÉFÉRENCES}

Allnut, V. (2012). Étude qualitative sur les attitudes des bibliothécaires québécois vis-à-vis la liberté intellectuelle et la censure. (Mémoire de maittrise inédit). Université de Montréal.

Leclercq, C. (2008). Les modalités de la prise en charge et de la gestion des événements traumatiques vécus par les policiers au cours de leurs fonctions au Québec (Thèse de doctorat inédite). Université de Montréal.

Blais, M. et Martineau, S. (2006). L'analyse inductive générale : Description d'une démarche visant à donner un sens à des données brutes. Recherches Qualitatives, 26(2), 1-18.

Bouchard, S. et Cyr, C. (2005). Recherche psychosociale : pour harmoniser recherche et pratique. Québec : Presses de l'Université du Québec. 761 p.

Brown, J. et Campbell, E. (1990). Sources of occupational stress in the police. Work et Stress, 4, 305-318.

Bruchon-Schweitzer, M. et Boujut, E. (2002). Psychologie de la santé : modèles, concepts et méthodes. Paris : Dunod.

Burke, R. (1993). Work-family stress, conflict, coping, and burnout in police officers. Stress Medicine, 9, 171-180.

Carlier, I., Lamberts, R. et Gersons, B. (2000). The dimensionality of trauma: A multidimensional scaling comparison of police officers with and without posttraumatic stress disorder. Psychiatry Research, 97, 29-39. 
Chermak, S., McGarrell, E. et Gruenewald, J. (2006). Media coverage of police misconduct and attitudes toward police. Policing: An International Journal of Police Strategies et Management, 29(2), 261-281.

Collins, P. A. et Gibbs, A. C. C. (2003). Stress in police officers: a study of the origins, prevalence and severity of stress-related symptoms within a county police force. Occupational Medicine, 53(4), 256-264.

Creswell, J. W., Hanson, W. E., Plano Clark, V. L. et Morales, A. (2007). Qualitative Research Designs: Selection and Implementation. The counseling psychologist, (35)2, 236-264.

Cyr, M.-P. (2010). La détresse psychologique et les facteurs professionnels : Étude du milieu policier. (Mémoire de maitrise inédit). Université de Montréal.

Deschamps, F., Pagnon-Badinier, I., Marchand, A.-C. et Merle, C. (2003). Sources and assessment of Occupational Stress in the Police. Journal of Occupational Health, 45, 358- 364.

Deschênes, A.-A, Desjardins, C. et Dussault, M. (2018). Psychosocial factors linked to the occupational psychological health of police officers: Preliminary study. Cogent Psychology, 5(1). https://doi.org/10.1080/23311908.2018.1426271

Desjardins, C. (2018). L'impact de l'aide psychologique spécialisée à la suite d'une exposition à un événement potentiellement traumatique sur la santé psychologique des policiers québécois. (Mémoire de maîtrise inédit). Université du Québec à Rimouski.

De Soir, E., Daubechies, F. et Van den Steene, P. (2012). Stress et trauma dans les services de police et de secours. Maklu Pub.

Drapeau, A., Marchand, A. et Beaulieu-Prévost, D. (2011). Epidemiology of psychological distress. Mental Illness. Understanding, Prediction, and Control, 4, 105-134. doi: 10.5772/30 872

Dumez, H. (2011). Qu'est-ce que la recherche qualitative? Le Libellio d'AEGIS, 7(4), 47-58.

Ellrich, K. et Baier, D. (2015). Post-Traumatic Stress Symptoms in Police Officers Following Violent Assaults: A Study on General and Police-Specific Risk and Protective Factors. Journal of Interpersonal Violence, 32(3), 331-356.

https://doi.org/10.1177/0886260515586358

Fortin M.-F., Gagnon J. (2016). Fondements et étapes du processus de recherche. Méthodes quantitatives et qualitatives. Montréal : Chenelière Éducation.

Gallant, R. 2018. Les facteurs de succès de la réintégration au travail d'un policier diagnostiqué avec un trouble de stress post-traumatique. (Mémoire de maîtrise inédit). Université du Québec à Rimouski.

Gavard-Perret, M.-L., Gotteland, D., Haon, C., et Jolibert, A. 2012. Méthodologie de la recherche en sciences de gestion : Réussir son mémoire ou sa thèse (2e édition). France : Pearson, 400 p.

Gilbert, M.-H. (2009). La santé psychologique au travail : conceptualisation, instrumentation et facteurs organisationnels de développement. (Thèse de doctorat inédite). Université de Montréal.

Gilbert, M.-H., Dagenais-Desmarais, V. et Savoie, A. (2011). Validation d'une mesure de santé psychologique au travail. Revue européenne de psychologie appliquée, 61, 195-203. https://doi.org/10.1016/j.erap.2011.09.001

Graziano, L., Schuck, A. et Martin, C. (2010). Police Misconduct, Media Coverage, and Public Perceptions of Racial Profiling: An Experiment. Justice Quarterly, 27(1), 52-76.

Hart, P. M., Wearing, A. J. et Headey, B. (1995). Police stress and well-being: Integrating personality, coping and daily-work experience. Journal of Occupational and Organizational Psychology, 68, 133-156. https://doi.org/10.1111/joop.1995.68.issue-2

Imbert, G. (2010). L'entretien semi-directif : à la frontière de la santé publique et de l'anthropologie. Recherche en soins infirmiers, 102(3), 23-34. doi:10.3917/rsi.102.0023

Institut universitaire en santé mentale de Montréal. (2018). En chiffres : au Québec. Repéré à http://www.iusmm.ca/hopital/santementale/en-chiffres.html

Kepplinger, H. M. (2007). Reciprocal effects: toward a theory of mass media effects on decision makers. The Harvard International Journal of Press/Politics, 12(2), 3-23. 
Kepplinger, H. M. et Glaab, S. (2007). Research Note: Reciprocal Effects of Negative Press Reports. European Journal of Communication, 22(3), 337-354.

Kepplinger, H. M. et Zerback, T. (2012). Direct and indirect effects of media coverage: Exploring effects of presumed media influence on judges, prosecutors, and defendants. Studies in Communication/Media, 1(3/4), 473-492.

Kop, N. et Euwema, M. (2001). Occupational stress and the use of force by dutch police officers. Criminal Justice and Behavior, 28(5), 631-652. https://doi.org/10.1177/009385480102800505

Kopel, H. et Friedman, M. (1997). Posttraumatic Symptoms in South African Police Exposed to Violence. Journal Of Traumatic Stress, 10(2), 307-317.

Korn, C. et Einwiller, S. (2013). Media coverage about organizations in critical situations: Analyzing the impact on employees. Corporate Communications : An International Journal, 18(4), 451-468.

Leclercq, C. (2008). Les modalités de la prise en charge et de la gestion des événements traumatiques vécus par les policiers au cours de leurs fonctions au Québec (Thèse de doctorat inédite). Université de Montréal.

Le mouvement santé mentale Québec. (2014). Pourquoi la santé mentale est-elle si importante pour les organisations? Repéré à http://www.mouvementsmq.ca/sante-mentale-et-travail

Lin, X., Lachlan, K. A., Spence, P. R. et Sellnow, T. L. (2016). Crisis communication, learning and responding: Best practices in social media. Computers in Human Behavior, 65, 601- 605.

Marchand, A., Boyer, R., Martin, M. et Nadeau, C. (2010). Facteurs prévisionnels du développement de l'état de stress posttraumatique à la suite d'un événement traumatique chez les policiers - Volet rétrospectif, rapport R-633, IRSST, 120 pages.

Massé, R., Poulin, C., Dassa, C., Lambert, J., Bélair, S. et Battaglini, A. (1998). The structure of mental health: higher-order confirmatory factor analyses of psychological distress and well- being measures. Social Indicators Research, 45, 475-504.

McCreary, D. R. et Thompson, M. M. (2006). Development of two reliable and valid measures of stressors in policing: The operational and organizational police stress questionnaires. International Journal of Stress Management, 13(4), 494. https://doi.org/10.1037/1072- 5245.13.4.494

Miller, J., Davis, R. C., Henderson, N. J., Markovic, J. et Ortiz, C. (2005). Measuring Influences on Public Opinion of the Police Using Time-Series Data: Results of a Pilot Study. Police Quarterly, 8(3), 394-401.

Moisseron, A. (2019). L'employeur face au maintien en emploi du salarié atteint de limitations fonctionnelles à la suite d'une lésion d'origine professionnelle : comparaison Québec et France. (Mémoire de maîtrise inédit). Université du Québec à Rimouski.

Morin, E. M. (2010). La santé mentale au travail : Une question de gros bon sens. Gestion, 35(3), 34-40. https://doi.org/10.3917/ riges.353.0034

Morin, E. M. (2008). Sens du travail, santé mentale au travail et engagement organisationnel, études et recherches, rapport R-543, IRSST (avec la collaboration de F. Aranha, FGV- EASP), 62 pages.

Morin, E. M. et Forest, J. (2007). Promouvoir la santé mentale au travail : donner un sens au travail. Gestion, 32(2), 31-36. doi:10.3917/riges.322.0031.

Paillé, P. et Mucchielli, A. (2010). L’analyse qualitative en sciences bumaines et sociales. 2e éd. Paris : Armand Colin.

Renck, B., Weisæth, L., et Skarbö, S. (2002). Stress reactions in police officers after a disaster rescue operation. Nordic Journal of Psychiatry, 56, 7-14. https://doi.org/10.1080/08039480252803855

Regehr, C., Johanis, D., Dimitropoulos, G., Bartram, C. et Hope, G. (2003). The Police Officer and the Public Inquiry: A Qualitative Inquiry into the Aftermath of Workplace Trauma. Brief Treatment and Crisis Intervention, 3(4), 383-396. doi:10.1093/brieftreatment/mhg030

Savoie, A., Brunet, L., Boudrias, J.-S. et Gilbert, M.-H. (2010). Surenchère de la non-santé psychologique au travail. Le Journal des Psychologues, 283, 31-34. https://doi.org/10.3917/jdp.283.0031 
Shane, J. M. (2010). Organizational stressors and police performance. Journal of Criminal Justice, 38, 807-818. https://doi.org/10.1016/j.jcrimjus.2010.05.008

St-Denis, K. (2018). La mobilisation des savoirs lors de recherches ethnographiques. Illustrations à partir de terrains chez les pompiers et paramédics du Québec. Approches inductives, 5(1), 64-86.

Stephens, C., Long, N. et Miller, I. (1997). The impact of trauma and social support on post- traumatic stress disorder in New Zealand police officers. Journal of Criminal Justice, 25, 303-313.

Sundaram, M. et Kumaran, M. (2012). A Study on Frequency of Occupational Stress among Grade II Police Constables. Asia Pacific Journal of Management et Entrepreneurship Research, 1(2), 134-144.

Tennant, C. (2001). Work-related stress and depressive disorders. Journal of Psychosomatic Research, 51, 697-704.

Théroux, M. (2000). Portrait et causes des abus d'autorité commis par les policiers québécois - Étude exploratoire. (Mémoire de maîtrise inédit). Université de Montréal.

Thomas, D. R. (2006). A general inductive approach for analyzing qualitative evaluation data. American Journal of Evaluation, 27(2), 237246. https://doi.org/10.1177/1098214005283748

Vasterman, P. L. M. (2005). Media-Hype : Self-Reinforcing News Waves, Journalistic Standards and the Construction of Social Problems. European Journal of Communication, 20(4), 508- 530.

Vasterman, P. L. M., Yzermans, C. J. et Dirkzwager, A. J. E. (2005). The Role of the Media and Media Hypes in the Aftermath of Disasters. Epidemiologic Reviews, 27(1), 107-114.

Veit, C. T. et Ware, J. E. (1983). The structure of psychological distress and well-being in general populations. Journal of Consulting and Clinical Psychology, 51(5), 730-742.

Violanti, J. M. (2010). Suicide or undetermined? A national assessment of police suicide death classification. International Journal of Emergency Mental Health, 12(2), 89-94. 